\title{
Das Technologie- und Gründerzentrum Wildau - eine Zwischenbilanz
}

\author{
Dr. rer. nat. Eberhard Brink
}

\section{Industriestandort Wildau}

Seit fast einhundert Jahren ist Wildau ein bedeutender Industriestandort vor den Toren Berlins. 1898 begann die Berliner Maschinenbau-Aktien-Gesellschaft - vormals L. Schwartzkopff, Berlin, in Wildau mit dem Bau einer Lokomotivfabrik, die am 1. September 1900 ihre Produktion aufnahm. Sie entwickelte sich schnell zu einem der führenden deutschen Schienenfahrzeugproduzenten. 600 bis 700 Lokomotiven für alle Spurweiten der Welt verließen pro Jahr das Werk (Abb. 1). Die bekannteste ist wohl die Schnellzuglokomotive der Baureihe 01 aus den dreißiger Jahren, die heute im Museum fuir Verkehr und Technik in Berlin zu besichtigen ist.

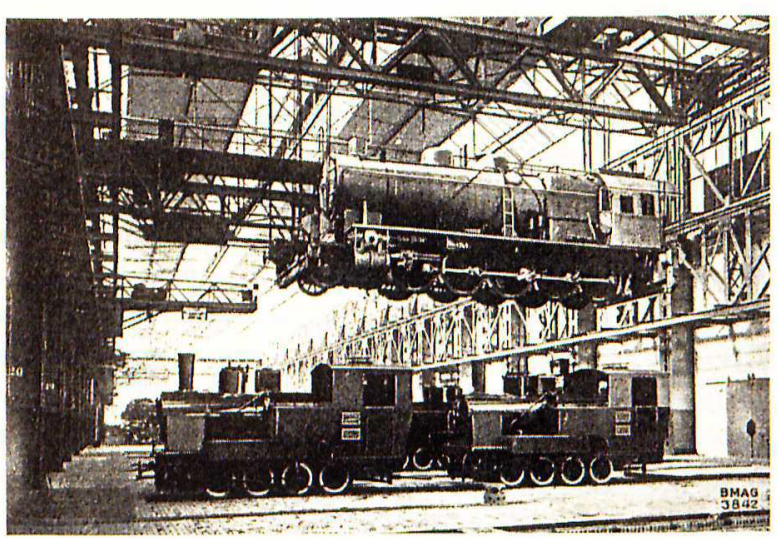

Abb. 1: Schwere 1E-Güterzüg-Lokomotive, am Krane hängend

1945 wurde das Unternehmen als ehemaliger Rüstungsproduzent enteignet. Die Mehrzahl der Produktionsanlagen ging nach Demontage an die Sowjetunion. Nach Gründung der DDR erfolgte der Wiederaufbau zum volkseigenen Schwermaschinenbau-Betrieb (SMB). Mit etwa 3.000 Beschäftigten war das Werk größter Arbeitgeber der Region und zugleich für den Kreis Königs Wusterhausen wirtschaftlich strukturbestimmend. Als Folge der tiefgreifenden politischen und gesellschaftlichen Veränderungen des Jahres 1990 wurde der Schwermaschinenbau Wildau als Treuhandunternehmen privatisiert und 1996 liquidiert. Zwei Tochterunternehmen - die Wildauer Kurbelwelle und der Sondermaschinenbau Wildau - setzen mit knapp 500 Beschäftigten die Tradition des Schwermaschinenbaus in Wildau fort. Bedeutende Entwicklungsimpulse für den Standort gehen von der 1991 neugegrïndeten Technischen Fachhochschule Wildau aus, die an die Tradtionen der früheren Ingenieurschule fuir Maschinenbau in Wildau ankniipft. 1995 verlegte sie ihren Hauptsitz auf das SMBGelände.
Als sich Anfang der neunziger Jahre die massiven Arbeitsplatzverluste im Wildauer Schwermaschinenbau abzeichneten, gehörte die Technische Fachhochschule Wildau gemeinsam mit dem Altkreis Königs Wusterhausen und früheren Mitarbeitern des Schwermaschinenbaus Wildau zu den Initiatoren für den Aufbau eines Technologie- und Griinderzentrums auf dem traditionsreichen Industriegelände. Die konzeptionellen Arbeiten wurden Ende 1994 mit der Grüindung der „Technologie- und Gründerzentrum Wildau GmbH“ durch den Landkreis Dahme-Spreewald, die Gemeinde Wildau, die Stadt Königs Wusterhausen und die Wirtschaftsförderungsgesellschaft des Landkreises als Gesellschafter, abgeschlossen.

Hinter diesen Bemühungen steckt die durch zahlreiche Beispiele gesicherte Erkenntnis, daß sich Technologie-, Gruinder- und Innovationszentren bei der Umstrukturierung und Revitalisierung von Industriestandorten bewährt haben. Deshalb wird der Aufbau des Wildauer Zentrums vom brandenburgischen Wirtschaftsministerium als prioritär im Landesinteresse eingestuft und ist für eine Förderung vorgesehen.

\section{Zielstellung und Zielgruppen}

Mit dem Aufbau des TGZ Wildau soll erreicht werden,

- innovative Unternehmen in Wildau anzusiedeln,

- die Arbeitsmarktchancen von qualifizierten Arbeitnehmern, insbesondere der Hochschulabsolventen, zu verbessern,

- einen traditionsreichen Industriestandort im Land Brandenburg, südöstlich von Berlin, der gegenwärtig zum großen Teil brachliegt, zu revitalisieren,

- im Landkreis Dahme-Spreewald einen zukunftsorientierten Wirtschaftsstandort zu entwickeln und

- den Technologietransfer aus der Technischen Fachhochschule Wildau in die Wirtschaft zu beschleunigen und

- die Ausbildung und Forschung an der Technischen Fachhochschule in der praktischen Zusammenarbeit mit den Unternehmen weiterzuentlickeln.

Zielgruppen des TGZ Wildau sind Existenzgründer, Jungunternehmer, Expertenteams aus Hochschulen, Forschungsinstituten oder aus Unternehmen, die

- im technologieorientierten Bereich tätig sind,

- die Vermarktung von innovativen Produkten und Leistungen in verschiedenen Technologiefeldern realisieren,

- an F\&E Aufgaben für die Wirtschaft arbeiten

- oder zeitlich befristete, wirtschaftlich orientierte Innovationsprojekte in die Praxis umzusetzen. 
Dazu gehören auch qualifizierte Dienstleister fuir technologieorientierte Unternehmen, wie z.B. auf dem Gebieten Arbeitsmedizin, Sicherheitstechnik, gewerblicher Rechtsschutz u.a.m.

Aus der gesamten Bundesrepublik und aus dem Ausland sollen Leistungsträger und Innovationspotentiale in die Region gezogen werden, um ihr spürbare wirtschaftliche Impulse zu vermitteln. Da das TGZ Wildau mit dem Ballungsraum Berlin infrastrukturell besonders eng verbunden ist, wird aus diesem großen Potential, wie schon gegenwärtig erkennbar, eine größere Zahl von Ansiedlern erwartet. Günstig ist die Nähe zum Flughafen Schönefeld als größtem Unternehmen der Region, der sich zu einem Kompetenzzentrum für Logistik und Verkehr entwickeln wird.

Das Zentrumsmanagement wird nach Umsetzung des Vorhabens den im TGZ angesiedelten Firmen interessante Serviceleistungen kostengiinstig anbieten, so zum Beispiel:

- einen zentralen Empfangs- und Betreuungsbereich für Besucher, Kunden und Gäste,

- Büro- und Sekretariatsdienste,

- Telekommunikationseinrichtungen/ Zugang zu Datenbanken,

- Kopierservice,

- Postdienst,

- Konferenzräume und Tagungsservice,

- Parkflächen für PKW.

Weitere Leistungen können über das vom TGZ aufgebaute Netzwerk von Kooperationspartnern schnell und günstig an die Firmen vermittelt werden. Das betrifft insbesondere die Nutzung der Verbindungen zur TFH Wildau, zur TINA Brandenburg, zur TVA Berlin, zur IHK Cottbus und zur WFG Dahme-Spreewald, um nur einige zu nemnen.

\section{Anforderungen an Ausbau und Ausstattung}

Die Eckpunkte hierfuir sind

- preisgünstige, zweckmäßige Räume für Firmen mit einer monatlichen Netto-Kaltmiete für Existenzgründer und Jungunternehmer von 8-12 DM/m²

- die befristeten Mietverhältnisse (in der Regel 5 Jahre mit einer Option auf max. 10 Jahre und Staffelmiete)

- kleinteilige Vermietung und Flexibilität im Hinblick auf den sich wandelnden Raumbedarf der Firmen während ihrer Mietzeit,

- multifunktionale Nutzungsmöglichkeiten des Raumangebots für wechselnde Unternehmen und deren sich verändernde Geschäftsfelder,

- Optionen für Ein-, Aus- und Umbau von Installationen und anderen Bauteilen nach den Anforderungen der Nutzer,

- die zukunftsorientierte Ausstattung mit Informations- und Kommunikationseinrichtungen (IuK),

- flexible, mieterspezifische Meßeinrichtungen für alle Medien, Versorgungs-, Entsorgungs- und anderen Dienstleistungen.

- die territorialen Gegebenheiten und Entwicklungsbedingungen des Standorts.

\section{Standort}

Wildau verfügt als Standort für ein Technologie- und Gründerzentrum uiber wichtige Vorteile, um im Wettbewerb der Ansiedlung von Existenzgründern und Jungunternehmern mit der wachsenden Zahl von Zentren, vor allem in Berlin, erfolgreich bestehen zu können:

- Wildau ist ein moderner, praxisorientierter Hochschulstandort mit beachtlichem Entwicklungspotential.

- Auf die äußerst vielfältigen Leistungsangebote der Metropole Berlin ist ein schneller Zugriff möglich.

- Die Verkehrsanbindung uiber Schiene und Straße ist sehr gut. Der Flughafen Schönefeld befindet sich in der Nähe.

- Eine denkmalgeschuitzte, fast einhundert Jahre alte Industriearchitektur bietet ein unverwechselbares Ambiente. Das weiträumige Industriegelände verfügt iiber genügend Expansionsmöglichkeiten für Unternehmen.

- Wildau und Umgebung besitzen eine hohe Wohnund Freizeitqualität.

- Vor allem im Süiden Berlins und im Norden des Landkreises ist ein geeignetes Arbeitskräftepotential vorhanden. In der Gemeinde Wildau haben sich 530 kleine und mittlere Unternehmen unterschiedlicher Branchen angesiedelt, darunter ein großer Teil aus dem Handwerk und dem produzierenden Gewerbe. Die Einwohnerzahl wächst deutlich, z. Z. beträgt sie mehr als 8.000 Einwohner, für das Jahr 2010 werden 13.000 vorausgesagt!

\section{Investitionsvorhaben}

Die TGZ Wildau GmbH konnte Ende Mai 1997 ein Grundstück von etwa $9000 \mathrm{~m}^{2}$ im Nordostbereich des Schwermaschinenbau-Geländes mit zwei alten Industriegebäuden (Geb. 42 und 43) (s. Abb. 2 und 3) erwerben.

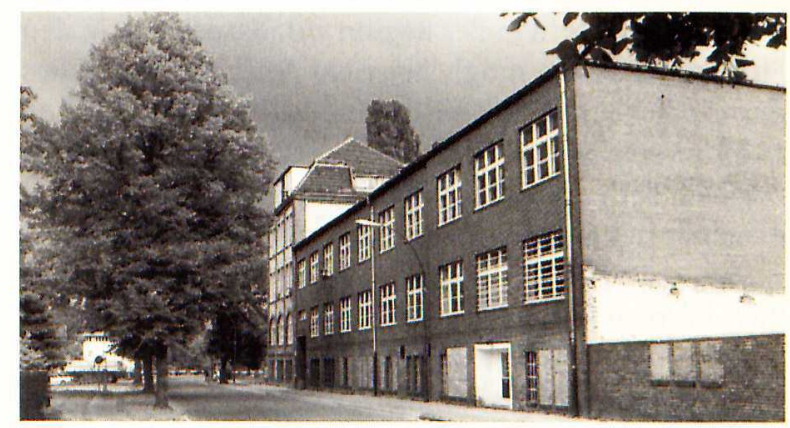

Abb. 2 und 3: Ehemalige Konstruktionsgebäude auf dem SMB-Gelände

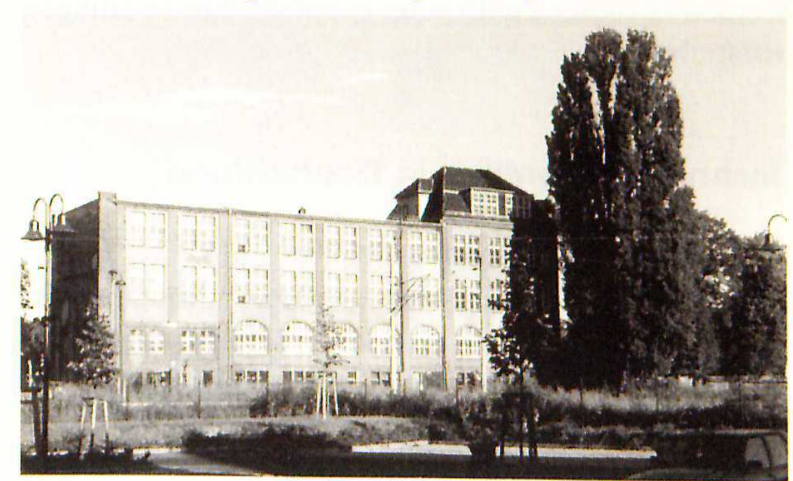


Die denkmalgeschuitzten Gebäude sollen bis zum Jahr 2000 zur Schaffung moderner Firmenmietflächen saniert und rekonstruiert werden. Dadurch werden etwa $3000 \mathrm{~m}^{2}$ Firmenmietfläche (FMF) geschaffen, uiberwiegend für Büros oder büroähnliche Nutzung, wie Kleinteile- und Gerätefertigung geeignet. Im Kellergeschoß ist die Aufstellung größerer Maschinen möglich. Ein Ergänzungsbau von $1.000 \mathrm{~m}^{2}$ vermietbarer Fläche soll das Raumangebot durch Labor- und Hallenflächen erweitern und flexibler machen.

Den Firmen werden moderne Gemeinschaftseinrichtungen (luK-Zentrum, Handbibliothek, elektronische Medienzugänge, Konferenz- und Beratungsräume) zur Verfuigung stehen. Im Kopfteil des Gebäudes 42 ist ein repräsentativer Empfangs-, Begegnungs- und Vermittlungsbereich vorgesehen.

Die Umgebung der bebauten Flächen wird durch gepflegten Alt- und Neubewuchs von regionalverträglichen Gehölzen und Anpflanzungen gestaltet, wodurch eine aufgelockerte, grüne Umgebung geschaffen wird. Die Altgebäude befinden sich unmittelbar an einer öffentlichen Straße und prägen in diesem Bereich das Bild des Ortes. Ausreichende und günstig gelegene Park-, Belade- und Entlademöglichkeiten für die Firmen und deren Kunden werden bereitstehen.

\section{Firmen}

Seit September 1995 betreut das TGZ Wildau die ersten Firmenansiedlungen, die durch Nutzung von Übergangslösungen auf dem SMB-Gelände möglich gemacht wurden. Nach Abschluß der gesamten Investition Schaffung von ca. $4000 \mathrm{~m}^{2}$ Firmenmietfläche in den Gebäuden $42 / 43$ und dem Neubau wird bei entsprechender Akzeptanz die Ansiedlung von 40-50 Unternehmen mit 200 bis 250 Mitarbeitern erwartet. Sekundäre Arbeitsplatzeffekte sind erfahrungsgemäß bei Zulieferern und Dienstleistern in der Region vorauszusagen. In den Firmen, zu denen möglichst viele Ausgründungen aus der Hochschule gehören sollten, werden Absolventen qualifizierte Arbeit finden. Ein TGZ vermag deshalb zugleich der gegenwärtig hohen HochschulabsolventenArbeitslosigkeit gegenzusteuern.

Zwei Wildauer TGZ-Firmen,

- DTK - Deutzer Technische Kohle GmbH und

- Dr. Alfred Schymalla, Ingenieurbetrieb für Wasserund Entsorgungstechnik

werden in dieser Publikation ebenfalls ihre Leistungen vorstellen.

\section{Technologiezentren in Deutschland}

Das erste deutsche Zentrum wurde Ende 1983 in Berlin (West) gegrïndet. Aktiver Partner der Gründung war die Technische Universität Berlin. Ein altes, brachliegendes Industriegebäude der AEG am Wedding wurde mit durch das Berliner Innovations- und Gründerzentrums (BIG) einer neuen Nutzung zugeführt. Fast gleichzeitig

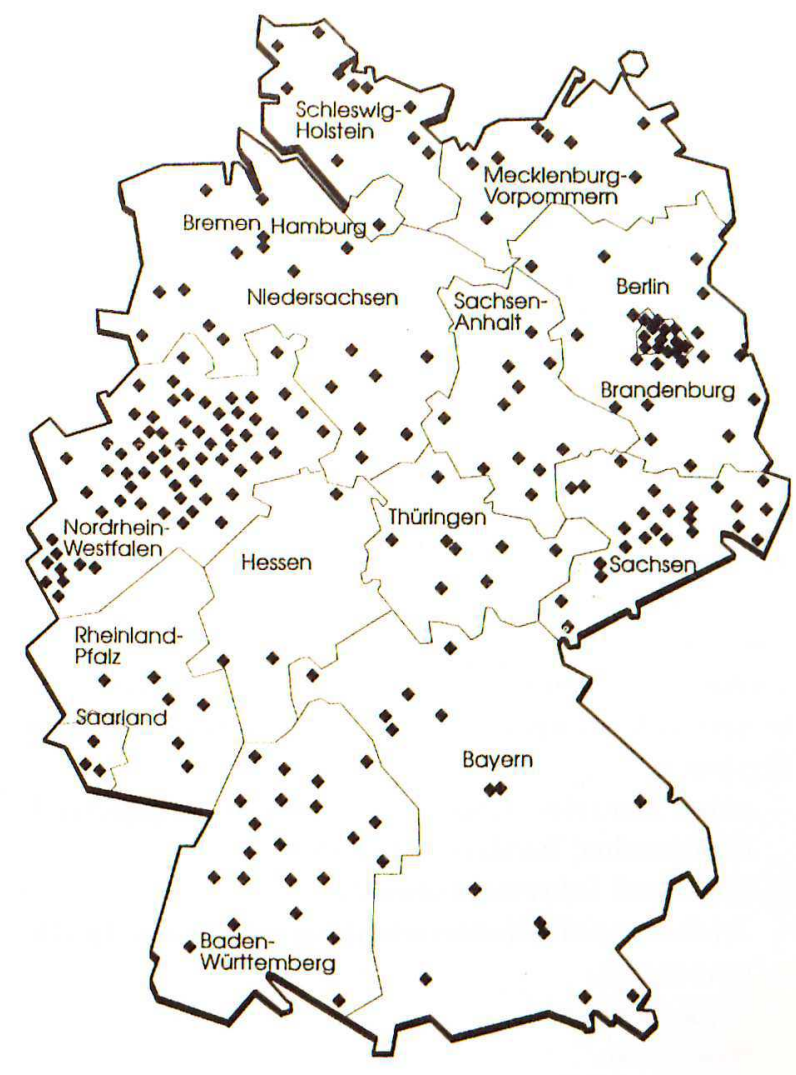

Abb. 4: Technologie- undGründerzentren in Deutschland 1996/97

entstanden an alten Industriestandorten in der Nähe von Technischen Hochschulen ähnliche Zentren in Nordrhein-Westfalen und Baden Württemberg. In Berlin (Ost) und auf dem Gebiet der neuen Bundesländer setzte die Entwicklung erst 1990 ein. Von den 200 Zentren in Deutschland befindet sich heute etwa ein Drittel in Ostdeutschland (s. Abb. 4). Die Zahl der Zentren wird in den nächsten Jahren weiter steigen. Für das Jahr 2000 werden 270 Zentren in Deutschland prognostiziert. Das Land Brandenburg fördert den Aufbau von 17 Zentren, zu denen auch das TGZ in Wildau gehört (s. Abb. 5). Hier wird es höchstens einen oder zwei neue Standorte geben. Doch werden sich einige Zentren durch Neubauten erheblich erweitern.

Berlin plant, die Zahl seiner Zentren von gegenwärtig 15 bis zum Jahr 2000 mindestens zu verdoppeln, verstärkt auch unter Einbeziehung privaten Kapitals.

In erster Linie scheint die Aufgabe von Technologie- und Griinderzentren darin zu bestehen,

- angemessene Firmenmieträume zu günstigen Konditionen anzubieten und

- profilkonforme Firmen anzusiedeln, um dadurch neue Arbeitsplätze zu schaffen.

Darauf beschränkt, wäre die Betreibergesellschaft nur ein „gehobener“ Entwickler und Verwalter einer speziellen Gewerbeimmobilie für besondere Zielgruppen von Unternehmen. Mit gegenwärtig 40.000 direkten $\mathrm{Ar}$ beitsplätzen in den Zentren sind die Arbeitsmarkteffekte beachtlich. Gelegentlich werden sie deshalb auch als „Jobmaschinen“ bezeichnet. Insgesamt sind das allerdings nur etwas mehr als $0,1 \%$ aller Arbeitsplätze in 


\begin{tabular}{|c|c|c|c|c|}
\hline $\begin{array}{l}\text { Technologle- und Gründer- } \\
\text { zentren in Brandenburg }\end{array}$ & $\left|\begin{array}{l}\text { zur Zoit } \\
\text { vermietbare } \\
\text { Nutzifl. } \\
\left(\mathrm{m}^{2}\right)\end{array}\right|$ & $\begin{array}{l}\text { Ausiast grad } \\
\text { der vermieatbaren } \\
\text { Nutzflächo } \\
\text { (\%) }\end{array}$ & $\mid \begin{array}{l}\text { Netto } \\
\text { Kaltmitate } \\
\left(\mathrm{DM} / \mathrm{m}^{2}\right)\end{array}$ & $\begin{array}{l}\text { Betriebskosten } \\
\text { voriuszanlung } \\
\text { (DM/m²) }\end{array}$ \\
\hline & & & & \\
\hline Technologiezentrum Tetrow GimbH & 6600 & 100,0 & $10,00-14,00$ & 6,80 \\
\hline $\begin{array}{l}\text { Business and Innovation Centre } \\
\text { Frankifurt (Oder) Gmbir (BIC) }\end{array}$ & 5500 & 78,2 & $12,00-17,00$ & 2,50 \\
\hline $\begin{array}{l}\text { Cotbuser Technologie- und Ent- } \\
\text { wicklungszentrum GmbH (CoTEC) }\end{array}$ & 10400 & 57.9 & $4,00-26,95$ & $2,50-4,50$ \\
\hline $\begin{array}{l}\text { Innovations- und Grunderzontrum } \\
\text { GmbH Ebarswalde (InnoZent) }\end{array}$ & 5500 & 90,6 & $5,00 \cdot 12,50$ & 5,00 \\
\hline $\begin{array}{l}\text { Strausberger Tochnologie- und } \\
\text { Innovationszentrum Gmbiti (STIC) }\end{array}$ & 7500 & 86.3 & 8.00 & 5,30 \\
\hline $\begin{array}{l}\text { Technologie- und } \\
\text { Gewerbezentrum Westprignitz GmbH (TOW) } \\
\text { Wittenberge }\end{array}$ & 820 & 85.9 & $5,00-12,00$ & 4,50 \\
\hline $\begin{array}{l}\text { Technologio- und Grunderzantrum } \\
\text { Gimbil der Region Uckermark. Schwedt }\end{array}$ & 700 & 70,7 & $9,00-10,00$ & 2,50 \\
\hline $\begin{array}{l}\text { Centrum fur Innovation \& } \\
\text { Technologie Guban-Zialona-Gora } \\
\text { GimbH (Cli) }\end{array}$ & $\begin{array}{l}z: 2.125 \\
\text { geplant } 4800\end{array}$ & 100,0 & $3,00-10,00$ & 3,00 \\
\hline $\begin{array}{l}\text { Technologita- und Gruinderzentrum } \\
\text { Eibe-Elster GmbH } \\
\text { Schieben/Finsterwalde/Bad Liebenwerca }\end{array}$ & 11000 & 72.7 & $3,00-10,00$ & 2,00 \\
\hline $\begin{array}{l}\text { Technologie- und Innovations- } \\
\text { zentrum Forstenwaida GmbH(TIF) }\end{array}$ & 1800 & 83,3 & 12.30 & 5,05 \\
\hline $\begin{array}{l}\text { Technologie- und Granderzantuum } \\
\text { Ostprignita-Ruppin Gmbit, Nouruppin }\end{array}$ & 3000 & 86,7 & $13,00 \cdot 15,00$ & 4,00 \\
\hline $\begin{array}{l}\text { Zentrum f. Entwicklung. } \\
\text { Innovation und Technologie in der } \\
\text { Niedertausitz Gmbit(Z.EI T). Schwarzheide }\end{array}$ & 600 & 100,0 & 5,00 & 4,00 \\
\hline Tochnologie- und Grunderzentrurn Wildau & $\begin{array}{r}\text { Z.Z. } 162 \\
\text { gepiant 4000 } \\
\end{array}$ & 100.0 & $\begin{array}{l}5,00-8,00 \\
8,00-12,00\end{array}$ & $\begin{array}{l}3,00 \\
4,00\end{array}$ \\
\hline $\begin{array}{l}\text { Technologiezentrum Verkehr } \\
\text { und Logistik Hennigsdorf GmbH }\end{array}$ & 4300 & 69,8 & 11,00 & 3.50 \\
\hline $\begin{array}{l}\text { Technologie- und Gronderzantrum "Flaming" } \\
\text { GmbH, Belzig }\end{array}$ & (im Auifbau) & & $a b 10,00$ & $2,50-4,00$ \\
\hline $\begin{array}{l}\text { Technoiogie- und Grunderzentrum im } \\
\text { Blotechnologiepark Luckenwaldo } \\
\text { GimbH }\end{array}$ & $\begin{array}{r}2500 \\
\text { (im Aúbaus) }\end{array}$ & & 8,00 & 5,00 \\
\hline $\begin{array}{l}\text { Tochnologiv- und Grinderzentrum } \\
\text { Brandensurg a.d.H. GmbH }\end{array}$ & $\operatorname{im}_{\text {Aufbau }}$ & & ca. 10.00 & ca. 5,00 \\
\hline
\end{tabular}

Abb.5: Technologie- und Gründerzentren im Land Brandenburg

Deutschland (indirekt geschaffene oder stabilisierte Arbeitsplätze im Umfeld der Zentren sind nicht eingerechnet). Da industrielle Vergangenheit und landespolitische Rahmenbedingungen wesentlichen Einfluß auf den Aufbau der Zentren haben, ist es nicht verwunderlich, daß es zwischen den Ländern deutliche Unterschiede gibt (Abb. 6).

Gemessen an dem Aufwand öffentlicher und privater Mittel für die Förderung von Technologiezentren wäre aber der Effekt zu gering, wenn er sich nur an der Zahl der Firmenansiedlungen und Arbeitsplätze messen ließe. Technologie- und Gründerzentren müssen vielmehr komplexe Innovationszentren sein, die in ihrem jeweiligen Umfeld Existenzgründungen und Technologietransfer fördern sowie wirtschaftsnahe Dienstleistungen anbieten. Sie entwickeln sich dann zu Keimzellen einer zukunftsorientierten wirtschaftlichen Entwicklung in der Region und leisten ihren spezifischen Beitrag beim Übergang der klassischen Industriegesellschaft zu einer modernen Dienstleistungs- und Informationsgesellschaft, in der auch innovatives, produzierendes Gewerbe vertreten ist. Sie beschleunigen den Aufbau eines modernen Mittelstandes durch Grüindungen neuer Unternehmen und durch ihren Beitrag zum Wachstum von kleinen Unternehmen nicht nur in Ostdeutschland, sondern iiberall dort, wo durch Abbau von Großunternehmen ein Strukturwandel stattfindet. Dadurch verschieben sich in den nächsten Jahren die regionalen Entwicklungsschwerpunkte für neue Zentren (Oberfranken,
Hessen). Unternehmensgründungen aus Hochschulen und außeruniversitären Forschungseinrichtungen kommt dabei eine besondere Bedeutung zu. Neuere Untersuchungen haben ergeben, daß es sich bei etwa zwei Drittel der TGZ-Unternehmen um akademische Ausgründungen handelt. Der Akademikeranteil bei den Beschäftigten liegt bei $50-60 \%$. Mehr als Dreiviertel der in TGZ angemeldeten Firmen sind technologieorientierte Unternehmen. Diese Entwicklung muß sich noch verstärken, nicht nur als Gegensteuerung in der gegenwärtig noch wachsenden Akademikerarbeitslosigkeit, die auch eine Folge des zunehmenden Arbeitsplatzabbaus in Großunternehmen und im Öffentlichen Dienst ist.

Als einem der wichtigsten Faktoren fuir den Erhalt der Wettbewerbsfähigkeit der Wirtschaft im internationaIen Maßstab müssen zielstrebig marktfähige Innovationen bei Produkten und Verfahren - Dienstleistungen eingeschlossen - gefördert werden. Technologiezentren können mit dazu beitragen, wenn sie durch Verknüpfung von Kompetenzen zu einer Drehscheibe des lebendigen Technologietransfers werden. Der Einsatz moderner Informations- und Kommunikationstechniken, die in jedem Technologiezentrum verfuigbar gemacht werden, erweitern deren Einflußbereich. Die Betreibergesellschaft in Verbindung mit ihren Partnern ist dann nicht nur Betreuer und Dienstleister für die „eigenen“ Firmen, sondern Multiplikator für die Wirtschaft mit wachsendem Einflußbereich.

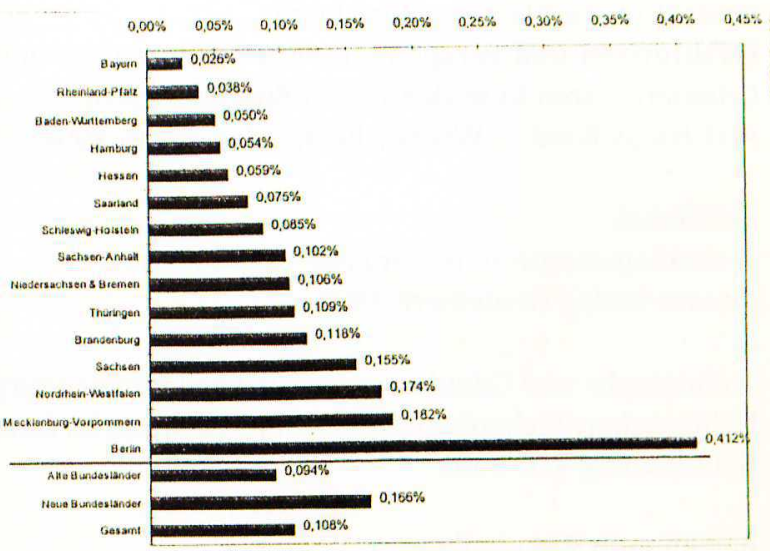

Abbl. 6 Anteil der TGZ-Beschäftigten an der Gesamtheit der Erwerbstättigen in den einzelnen Bundesländern

Der wichtigste Partner eines Technologiezentrums, das den Namen zu Recht tragen will, ist eine Hochschuleinrichtung. Gerade die unmittelbare Nachbarschaft zu einer Technischen Fachhochschule ist ein bedeutender Erfolgsfaktor fuir die zeitgemäße Aufgabenerfüllung eines Technologie- und Gründerzentrums. Diese Partnerschaft hat mit Sicherheit auch positive Ruickwirkungen auf Lehrende und Studierende an der Hochschule, nicht zuletzt auch auf ihre Struktur.

Je besser Technologiezentren diese anspruchsvollen Aufgaben bewältigen, umso schneller werden Skepsis und Kritik, die gelegentlich in die öffentliche Diskussion gebracht worden sind, durch sachbezogene, keineswegs selbstzufriedene Argumente entkräftet werden. 


\section{Ausgewålhlte Literatur}

G. Baranowski, B. Groß (Herausgeber) Innovationszentren in Deutschland 1996/97 Weidler Buchverlag Berlin 1996

B.Groß (Herausgeber)

Technologie- und Gründerzentren in Deutschland und ihr Beitrag zur Entwicklung technologie-orientierter Unternehmen.

Studie Februar 1997

Arbeitsgemeinschaft Deutscher Technologie- und Gründerzentren e.V.

R. Stemberg u. a.

Bilanz eines Booms.

Wirkungsanalyse von Technologie- und Gründerzentren in Deutschland.

Dortmunder Vertrieb für Bau- und Planungsliteratur, Dortmund 1996

8. Internationale ADT-Jahreskonferenz 1996 Perspektiven für Existenzgründung, Kooperation und Wachstum von Technologieunternehmen NICE - Networks of Innovation Centres in Europe Berlin, 7. - 9. November 1996, Konferenzmaterialien Arbeitsgemeinschaft Deutscher Technologie- und Gründerzentren ADT e.V.

B.Groß (Herausgeber) Innovationszentren der 90er Jahre.

Erfahrungen und Perspektiven der Technologie- und Gründerzentren in in den neuen Bundesländern.

ADT-Focus Band 7, Weidler Buchverlag Berlin 1994

\section{F. Pleschak}

Technologiezentren in den neuen Bundesländern Physica-Verlag Heidelberg 1995

Technologie- und Gründerzentren im Land Brandenburg Herausgeber: Technologie- und Grüinderzentren im Land Brandenburg e.V. 1996

Regionen in Brandenburg Wirtschaftsregion Dahme-Spreewald Image-Broschiire des Landkreises und der Wirtschaftsförderungsgesellschaft Dahme-Spreewald 1996

\section{Synergiejournal}

Technologiezentren: Drehscheiben des Transfers

Technologiezentren im Land Nordrhein-Westfalen e.V. März 1997

TGZ Wildau, Konzeption fuir den Aufbau und Betrieb, erstellt durch: WFG Wirtschaftsförderungsgesellschaft Dahme-Spreewald 1994

75 Jahre Schwartzkopff Industrie-Bibliothek, Die illustrierte Zeitschrift der deutschen Wirtschaft

Band 23, Berlin 1928

\section{Verfasser}

Dr. rer. nat. Eberhard Brink, Geschäftsführer Technologie- und Gründerzentrum Wildau GmbH Bahnhofstr. 1 (SMB-Gelände, Haus 13) 15745 Wildau Tel.: (03375) 508294 